\title{
Cantor devant la Loi
}

Henri Volken

\section{OpenEdition \\ Journals}

Édition électronique

URL : http://journals.openedition.org/ress/465

DOI : $10.4000 /$ ress.465

ISSN : 1663-4446

\section{Éditeur}

Librairie Droz

Édition imprimée

Date de publication : 1 janvier 2006

Pagination : 77-88

ISBN : 2-600-00959-0

ISSN : 0048-8046

Référence électronique

Henri Volken, «Cantor devant la Loi », Revue européenne des sciences sociales [En ligne],

XLIV-133 | 2006, mis en ligne le 12 novembre 2009, consulté le 03 mai 2019. URL : http://

journals.openedition.org/ress/465; DOI : 10.4000/ress.465 


\section{Henri VOLKEN}

\section{CANTOR DEVANT LA LOI}

\section{LA LOI ET LE DIVIN}

Hors de son contexte, la nouvelle Devant la loi offre la vision d'une quête un peu absurde, très probablement vouée à l'échec, d'un homme de la campagne désireux d'entrer dans la «loi». Rien ne permet au lecteur de connaître les raisons de cette aspiration, ni de savoir en quoi consiste cette «loi». Kafka nous montre un être quelque peu naïf au début du récit, puis étonné de la résistance du gardien qui l'empêche d'entrer mais n'exclut pas la possibilité d'une permission ultérieure. L'homme pense pourtant que la loi doit être «accessible à tous et tout le temps ». Puis au fur et à mesure que les difficultés s'accumulent, l'attention de l'homme se détourne de la loi pour se fixer sur le gardien. Les détails du visage et des vêtements de celui-ci deviennent le pôle attracteur de l'intérêt de l'homme, et la loi, bien que produisant une «glorieuse lumière», semble plutôt lui fournir en arrière-fond un prétexte à continuer son effort et à justifier sa démarche. La recherche d'une entrée dans la loi devient apparemment son unique raison de vivre, mais semble en même temps prendre une forme de moins en moins réelle. Le sens de la quête paraît se réduire à la quête elle-même. L'homme ne bouge plus de la porte devant la loi, perd progressivement ses moyens et finit par mourir. Avant sa mort, il apprend cependant que cette porte n'était destinée qu'à lui et que le gardien va la fermer et s'en aller. Une pirouette finale assortie d'une pointe d'humour noir, qui laisse le lecteur perplexe, aux prises avec de nombreuses interrogations.

Une des questions essentielles que le lecteur se pose, concerne l'objet central de la nouvelle. L'auteur ne précise pas - et rien ne permet de le découvrir - à quoi fait allusion cette «loi » qui se trouve à l'origine de la quête obstinée de l'homme de la campagne, expression que Kafka utilise d'ailleurs de préférence pour désigner une personne peu instruite, un peu lourde et soumise. La dimension cryptique de l'utilisation du concept de «loi » favorise les interprétations les plus diverses. Les lecteurs juristes tenteront probablement de rester le plus près possible de l'acception évidente du terme et y verront peut-être une idéalisation, une abstraction de la loi, ou des lois, dans le sens de la science du droit. Les philosophes y liront peut-être une recherche du sens de la vie, ou la quête de l'absolu. Et plus généralement, chaque lecteur y verra le reflet de ce qui lui paraît être l'objet le plus digne d'une quête aussi entêtée.

Parmi toutes ces interprétations on trouve entre autres - en partie suggérées par quelques éléments biographiques de l'auteur - des approches théologiques qui lient le terme de loi à celui de Dieu. Et sans vouloir en faire une équation au sens 
mathématique, on peut évoquer la relation étroite reliant les deux termes «loi Dieu» qui transparaît clairement dans divers contextes de l'histoire des religions. Dans le judaïsme par exemple ces deux concepts sont souvent associés de manière très étroite, parfois proche de l'identification ${ }^{1}$. Et dans les textes bibliques, non seulement dans la Thora, on trouve de nombreux exemples où la «parole» divine peut-être interprétée comme la «loi » divine. La loi est alors associée de manière plus ou moins forte à l'essence de Dieu. Selon l'évangile de Jean par exemple, «la parole était avec Dieu, et la parole était Dieu $»^{2}$. La quête dont traite la nouvelle de Kafka peut être vue sous cet éclairage, et dans un sens très large, comme une recherche de Dieu. Cela n'est bien sûr qu'une spéculation, une tentative herméneutique parmi d'autres, bien que quelques indices semblent autoriser une interprétation dans ce sens. Dans son journal, Kafka décrit sa nouvelle comme «légende», parfois comme «parabole», ce qui pourrait suggérer effectivement la possibilité d'une connotation religieuse. Sans parler de la «glorieuse lumière» déjà évoquée. Mais c'est surtout en replaçant la nouvelle dans son contexte, le roman Le procès, que cette piste devient plausible. Le texte de la nouvelle apparaît dans le chapitre 9 du livre, le chapitre dit «du dôme», où il fait l'objet d'une véritable «exégèse» de la part de l'abbé. Le contexte solennel et la présence essentielle d'un homme d'église, suggère la double appartenance de la nouvelle au monde juridique et au monde religieux. L'impression d'ensemble créé par le décor, l'habit ecclésiastique et la terminologie spécifique utilisée dans l'argumentation, rend l'interprétation théologique admissible.

Il faut se souvenir aussi que la nouvelle a été écrite vers la fin de la période allant de 1910 à 1920, c'est-à-dire dans les années où Kafka - issu d'un milieu juif peu pratiquant $-\mathrm{s}$ 'est replongé dans le judaïsme et la culture juive, et qu'il a fait la connaissance de la personne et de l'œuvre du philosophe de la religion, Martin Buber. C'est à cette époque qu'il s'est intéressé à la tradition religieuse juive, au Talmud et à la Kabbale en particulier. Tout cela pourrait être l'indice d'un sens caché de la nouvelle, bien qu'il n'y ait aucune preuve permettant d'affirmer ce lien avec certitude, comme nous l'avons déjà dit.

Le dialogue entre l'abbé et Joseph K. dans le dôme, tourne autour des interprétations possibles de la «parabole» et anticipe les préoccupations légitimes de ses lecteurs. Quel est le sens de la quête obstinée de l'homme de la campagne? La nouvelle est-elle le récit d'un échec, ou d'une démarche dont le sens réside dans la quête elle-même? L'exégèse s'engage dans de nombreuses directions et ouvre d'innombrables pistes d'interprétation. Mais la longue suite d'arguments et contre arguments à propos de son sens, finit par révéler la présence d'une ironie grinçante, burlesque, un peu désespérée, aboutissant en fin de compte à un vertige dialectique: Le raisonnement ne peut pas avoir de fin. Le problème de l'interprétation de la nouvelle ne pourra jamais être décidé ${ }^{3}$.

Ne pas avoir de fin: c'est exactement l'une des propriétés majeures, un des aspects terrifiants de la barrière invisible qui se dresse entre l'homme de la

\footnotetext{
Im Judentum ist Gott Gesetz geworden, im Christentum Mensch. (Rupert Lay)

Jean 1,1

3 Heinz Politzer a comparé la nouvelle à un test de Rorschach: toute interprétation du texte en dit plus long sur la personne qui l'entreprend, que sur le texte lui-même.
} 
campagne et la loi. C'est ce qui constitue probablement l'essence secrète de sa peur. Le gardien l'avertit:

\begin{abstract}
Essaie donc d'entrer d'entrer malgré ma défense. Mais retiens ceci: je suis puissant. Et je ne suis que le dernier [vu de l'extérieur: le premier] des gardiens. Devant chaque salle il $\mathrm{y}$ a des gardiens de plus en plus puissants, je ne puis même pas supporter l'aspect du troisième après moi.
\end{abstract}

Le gardien n'exclut donc nullement la possibilité que l'homme pénètre un jour dans la loi jusqu'à une certaine profondeur, mais rappelle cette menace itérative, qu'il y aura toujours un nouveau gardien, encore plus puissant, et qui s'opposera à l'intrus à chaque étape. Ce qui implique en particulier qu'il ne peut pas exister de dernier gardien! L'entreprise de l'homme de la campagne est donc condamnée, à l'échelle humaine en tout cas, et la quête n'aboutira jamais. La «loi » possède une structure d'infini!

Notre lecture du texte possède donc une composante «théologique» et une composante structurelle. Peut-on concilier les deux? Peut-on, ou doit-on rapprocher Dieu et l'infini?

\title{
LE DIVIN ET L'INFINI
}

Or précisément, la théologie chrétienne, en tous cas depuis Saint Augustin (354-430), dans une démarche tendant à capter l'idée d'absolu dans une terminologie suggestive, associe très clairement l'idée de Dieu à la notion d'infini. Dans sa «Cité de Dieu» on trouve par exemple au chapitre XVIII la remarque:

«Quant à ce qu'ils disent, que Dieu même ne saurait comprendre des choses infinies, il ne leur reste plus qu'à soutenir, pour mettre le comble à leur impiété, qu'il ne connaît pas tous les nombres.»

Il s'agit d'une allusion directe au dualisme des notions d' «infini actuel» par opposition à la notion d' «infini potentiel». Nous allons revenir plus loin sur cette distinction essentielle dont la caractérisation terminologique remonte à Aristote, bien que l'idée soit bien plus ancienne. Pour l'instant notons simplement que la théologie chrétienne du moyen âge place l'infini et sa maîtrise au rang des attributs essentiels de Dieu. Au XIII ${ }^{\mathrm{e}}$ siècle Thomas d'Aquin (1227-1274) a avancé l'idée plus précise encore que Dieu est infini ${ }^{4}$. L'infini devient alors, dans une grande partie de l'argumentation théologique chrétienne, une qualité essentiellement divine, inaccessible par nature à l'homme. Mais cet infini, dont Thomas admet l'existence reste essentiellement un infini en puissance, même si certaines de ses affirmations laissent parfois entrevoir la possibilité d'un infini en acte.

Le changement radical intervient avec Duns Scot (1265-1308) pour lequel l'infini actuel non seulement est parfaitement pensable, mais encore d'essence positive. Bien sûr, l'infini actuel, en tant que composante de l'être, est réservé à Dieu. On est encore loin d'accorder un statut ontologique positif au concept laïque de l'infini actuel comme on le verra dans le monde mathématique de la deuxième moitié du XIX ${ }^{\mathrm{e}}$ siècle.

${ }^{4}$ «Dieu, dont l'être est infini, ...» dans: Somme Théologique, première partie. 
Dans la culture judaïque, on trouve des traces d'une approche encore plus systématique. En particulier dans le contexte mystique de la Kabbale, l'infini prend une importance capitale. On trouve dans la Kabbale dix éléments secrets, les Sefirot, symbolisés par des sphères formant un espace multi dimensionnel d'une grande complexité, et qui représentent des ensembles d'attributs divins. Une savante combinatoire les relie aux différentes facettes de Dieu. Mais celui-ci se trouve caché derrière les Sefirot, s'étendant sans limites. A cette entité divine, illimitée, la Kabbale donne le nom de Ein Sof, l'infini. Ce contexte constitue l'un des exemples manifestes où l'on voit la forte proximité sémantique entre les concepts de Dieu et de l'infini.

Si nous revenons à la nouvelle de Kafka, et quelle que soit l'interprétation que nous donnions au concept de loi, la structure itérative - un gardien suivi d'un autre - utilisée dans le texte pour décrire la loi, renvoie clairement à la notion d'infini. Kafka utilise cette structure sans doute parce qu'elle implique une double impossibilité. Impossibilité de se rapprocher de la loi, à fortiori de l'atteindre, mais aussi impossibilité aussi de la saisir globalement comme entité. Nous pouvons illustrer très simplement ces deux difficultés dans un exemple simple, à l'aide des nombres naturels, qui constituent un modèle familier de l'infini. En partant de 0 et en ajoutant 1 à chaque pas, nous avançons aussi loin que nous voulons dans les nombres, mais sans jamais nous rapprocher de l'infini; la distance à l'infini reste la même à chaque étape. D'autre part, si nous pouvons concevoir et atteindre des nombres aussi grands que nous voulons, nous ne pouvons jamais ${ }^{5}$ percevoir l'ensemble de tous les nombres comme une unité qui les contiendrait tous. Car cette unité constituerait en elle-même un exemple de nombre infini, et reste donc inaccessible à notre entendement aussi longtemps que nous conservons une approche naïve des nombres. Le concept d'infini actuel pose ces mêmes problèmes, mais en toute généralité.

Nous allons élargir notre point de vue et brièvement retracer quelques étapes de l'histoire de l'infini dans la pensée et la civilisation occidentales en dehors du contexte religieux.

\section{L'INFINI DEVANT L'HISTOIRE}

L'une des premières utilisations systématiques de la notion d'infini comme principe explicatif - du moins dans la culture occidentale - se trouve probablement chez les Présocratiques et plus exactement dans la pensée et l'enseignement d'Anaximandre ( 610 - 546). Pour ce philosophe, l'apeiron, l' in-fini, est le principe et la base de tout. L'infini est notamment à l'origine de tous les êtres, qui sont nécessairement finis, et de leur devenir. Et ce n'est que par lui que le monde devient compréhensible:

Anaximandre a dit que l'Infini est le principe des choses qui sont (...) Ce dont la génération procède pour les choses qui sont, est aussi ce vers quoi elles retournent sous l'effet de la corruption, selon la nécessité; car elles se rendent mutuellement justice et réparent leurs injustices selon l'ordre du temps.

(Simplicius, Commentaire sur la Physique d'Aristote, 24,13)

\footnotetext{
Dans une première approche, sans le recours au formalisme mathématique introduit par Cantor.
} 
Mais l'appréciation dans un sens positif de l'infini que l'on voit chez Anaximandre, reste isolée dans la philosophie grecque. A l'exception peut-être d'Empédocle, d'Anaxagore ou des Atomistes, la notion d'apeiron est majoritairement considérée chez les penseurs grecs comme négative. Elle représente en général quelque chose de non fini, de non déterminé, de non achevé et ne peut expliquer, la plupart du temps, que les aspects imparfaits, mauvais des choses. Les Pythagoriciens par exemple identifient ce qui est limité, mesurable et exprimable par des nombres (entiers), avec le beau, l'utile et le bon. L'infini au contraire pour eux est à l'origine de la découverte de l'incommensurabilité, donc de l'existence des nombres irrationnels, incompatibles avec la doctrine pythagoricienne. Bref, l'infini représente le mal dans un sens très large.

Empédocle et les Atomistes admettent l'infini dans l'ordre macroscopique, mais le rejettent dans l'ordre microscopique: la nature n'est pas divisible à l'infini. Zénon ( 495 - 445) utilise l'infini dans le but de soutenir les thèses de son maître Parménide, qui soutient que le mouvement et la multiplicité sont impossibles. Pour cela, le concept d'apeiron permet à Zénon d'introduire des paradoxes censés démontrer cette thèse dans un bel exemple d'argumentation par l'absurde.

Platon ( 428 - 348) partage avec les Pythagoriciens le jugement positif de la notion de limitation, de finitude, qui seules peuvent être à l'origine de la bonté et de la beauté, bien qu'il admette que l'interaction dynamique fini - infini puisse être à l'origine de la création de la perfection.

Eudoxe ( 406 - 355), mathématicien et astronome disciple de Platon, s'appuie sur le fameux principe d'Archimède:

En soustrayant de la plus grande de deux grandeurs données plus de sa moitié, et du reste plus de sa moitié, et ainsi de suite, on obtiendra [en un nombre fini d'étapes] une grandeur moindre que la plus petite

pour créer sa subtile méthode de l'exhaustion qui lui permet d'introduire des idées que l'on retrouvera plus tard dans les discussions sur les débuts de l'analyse. Mais sa méthode, et sa théorie des proportions, lui permet surtout d'éviter les discussions délicates autour de la divisibilité à l'infini, et de ce qu'on appellera plus tard les infiniment petits. Il peut ainsi parler du continu sans avoir besoin d'aborder explicitement la notion d'infini.

Mais c'est à Aristote ( 384 - 322), qui s'est penché longuement sur la question de l'infini, que l'on doit la distinction infini potentiel - infini actuel. Cette distinction, qui reflète l'ambiguïté de la position d'Aristote, est à l'origine de nombreuses controverses et disputes et s'est conservée partiellement jusqu'à nos jours. Aristote s'oppose fortement à la possibilité de considérer l'infini comme un principe ou une substance, même considérés sous un aspect négatif. Cette opposition se fait malgré la bienveillance envers cette idée qu'il croit déceler chez les Pythagoriciens et chez Platon. Il admet cependant l'infini comme possibilité. Pour lui, dont l'approche naturaliste et expérimentale préfigure la démarche scientifique que nous connaissons aujourd'hui, les opérations d'addition et de subdivision peuvent ne pas être limitées. Dans le domaine des nombres par exemple, il est possible de rajouter une unité à n'importe quel nombre sans être jamais arrêté, de même qu'il est possible de diviser un segment en parties plus petites. Mais jamais la totalité des nombres ou des segments divisés n'a d'existence propre. Cette 
totalité ne peut pas constituer un concept aux yeux d'Aristote. La citation suivante peut résumer sa position ambivalente:

Il est clair que l'infini existe dans un sens, et, en un autre sens, il n'existe pas... ${ }^{6}$

La distinction infini potentiel - infini actuel restera l'objet de discussions passionnées, aussi bien dans le domaine de l'épistémologie et des mathématiques que de la théologie. Nous allons esquisser très sommairement quelques étapes décrivant l'approche mathématique plus récente, celle qui a permis les avancées spectaculaires dans notre compréhension de l'infini. Il faut citer quelques précurseurs du moyen âge, parlant de l'infini dans un sens non exclusivement théologique, comme l'érudit anglais Robert Grosseteste (1170-1253) qui, l'un des premiers probablement, affirme qu'un nombre peut-être infini et surtout, six siècles avant Cantor, qu'un nombre infini peut être plus grand qu'un autre. Grégoire de Rimini (1300-1358) soutient explicitement l'existence de l'infini actuel et parle lui aussi d'infinis inégaux.

Galilée (1564-1642), à la suite de Giordano Bruno, semble lui aussi avoir admis l'existence de l'infini dans sa forme actuelle, bien que ses formulations restent prudentes, probablement à cause de l'Inquisition qui l'avait déjà condamné pour ses idées et qui le surveillait étroitement. Mais la discussion dans ses Dialogues des sciences nouvelles (1638) décrivent pour la première fois l'une des caractéristiques des ensembles infinis, qui sera reprise deux siècles plus tard par Dedekind et Cantor. Il s'agit du fait qu'il y a autant de nombres carrés qu'il y a de nombres ${ }^{7}$. Ce principe est fondamental pour la suite et il vaut la peine de lire l'extrait des Dialogues ${ }^{8}$ qui l'établit:

$\begin{array}{ll}\text { Salviati } & \begin{array}{l}\text {...Donc si je dis que tous les nombres, carrés et non carrés, sont plus que les } \\ \text { carrés seuls, j'énonce une proposition très véritable. Sommes-nous d'ac- } \\ \text { cord? }\end{array} \\ \text { Simplicio } & \text { Il est impossible de ne pas l'être. } \\ \text { Salviati } & \begin{array}{l}\text { Si maintenant je demande combien il y a de nombres carrés, on pourra } \\ \text { répondre [...] qu'ils sont aussi nombreux que leurs propres racines [...]. }\end{array} \\ \text { Simplicio } & \text { C'est ainsi. } \\ \text { Salviati } & \begin{array}{l}\text { Mais si j'en arrive à demander combien il y a de racines, on sera forcé de } \\ \text { répondre qu'il y en y autant que de nombres, car il n'est pas un nombre qui } \\ \text { ne soit racine de quelque carré; cela étant nous pouvons affirmer que les } \\ \text { nombres carrés sont autant que tous les nombres, puisqu'ils sont autant que } \\ \text { leurs racines, et que tous les nombres sont racines. Cependant, nous disions, } \\ \text { pour commencer qu'il y a plus de nombres en tout qu'il n’y a, en tout de } \\ \text { carrés, la majeure partie des nombres étant non carrés. [...] Et pourtant dans } \\ \text { le nombre infini, si nous étions à même de le concevoir, nous devrions } \\ \text { reconnaître que les carrés sont autant que tous les nombres. }\end{array}\end{array}$

6 Cité dans [L]. Cf références.

7 Il s'agit ici de nombres naturels.

\& Galilée, Dialogues, Collection Histoire de la pensée, Hermann, 1997 
Galilée met en évidence l'une des particularités des mécanismes de l'infini et ouvre la porte à une approche plus mathématique et épistémologique de cette notion.

Par la suite, les discussions sur l'infini se reportent dans un premier temps sur l'infiniment petit, poursuivant en cela des idées des Atomistes, d'Eudoxe, d'Archimède, mais aussi des suggestions plus récentes, également proposées par Galilée, concernant la circonférence d'une roue. Bonaventure Cavalieri (15981647) développe une géométrie basée sur les infiniment petits - les indivisibles. Pascal et Fermat systématisent les idées de Cavalieri et les rendent plus acceptables. John Wallis prépare, par son apport à la méthode des indivisibles, la création de l'analyse moderne - notre calcul différentiel et intégral - par Newton et Leibniz. Le problème n'est plus la définition de nombres infinis, mais le statut et les mécanismes de grandeurs infiniment petites.

C'est le prêtre tchèque Bernhard Bolzano (1781-1848), rejeté par le clergé pour ses idées révolutionnaires, qui reprend deux siècles plus tard, le fil des réflexions de Galilée sur l'infini. En particulier, il montre, en adoptant une définition nouvelle de la notion de fonction, comment étendre la découverte de Galilée à des ensembles de nombres réels: il y a autant de points dans l'intervalle $[0,1]$ que dans l'intervalle $[0,2]$ qui pourtant possède une longueur double. Il réunit ses idées et découvertes sur l'infini dans un livre qui passe presque inaperçu: Paradoxien des Unendlichen (1850), mais qui retiendra l'attention de quelques rares mathématiciens.

\section{CANTOR DEVANT L'INFINI}

Mais l'infini actuel n'a été réellement admis comme objet d'étude à part entière que depuis les travaux de Georg Cantor (1845-1918). Il a fallu une approche mathématique radicalement nouvelle pour intégrer pleinement l'infini dans le champ des connaissances scientifiques. Cela ne s'est pas passé sans problèmes. Les réactions de rejet et les oppositions multiples de la part d'un certain nombre de ses collègues lui ont rendu la vie difficile et ont empêché Cantor d'obtenir la reconnaissance et la carrière académique que son coup de force intellectuel auraient dû lui procurer.

La clé pour la compréhension de la notion d'infini actuel a été découverte par Cantor après un changement décisif du point de vue courant. Au lieu de situer l'exploration de l'infini mathématique au niveau des nombres (ou des points, s'il s'agit de considérations géométriques), Cantor propose d'utiliser systématiquement le concept d'ensemble et de redéfinir les nombres dans cet univers. Ses premiers travaux, qui concernent la notion topologique de points limites, l'amènent progressivement à entrevoir la possibilité de construire et de manipuler des nombres infinis et de les utiliser pour décrire la structure nouvelle et extraordinairement complexe de l'infini qu'il y perçoit.

C'est à travers une série de paradoxes que Cantor découvre la vraie nature de l'infini. Il montre en 1877 que des objets géométriques de dimensions différentes, un segment de droite, un carré dans le plan ou un cube, ont tous le «même nombre », le même cardinal dans sa terminologie, - un nombre infini - de points. Cela avait été anticipé par Bolzano dans son utilisation d'une nouvelle conception 
de la notion de fonction La dimension de l'objet ne joue aucun rôle dans ces considérations! Cela constitue une vérité difficile à admettre pour un mathématicien de cette époque, et Cantor lui-même a longuement tenté de démontrer le contraire. Mais, poussé par la preuve irréfutable qu'il découvre, il se lance dans l'étude analytique de l'infini avec les nouveaux moyens qu'il développe. Cela lui vaut l'inimitié d'un grand nombre de ses collègues et notamment Leopold Kronecker pour qui seul les nombres naturels existent et qui refuse d'admettre l'existence de nombres irrationnels et s'oppose violemment contre toute idée d'utiliser l'infini dans la discussion mathématique autrement que sous forme de métaphore.

Un autre des paradoxes mis en évidence par Cantor s'apparente à la découverte étonnante de Galilée dont nous avons parlé: il y a autant de nombres pairs qu'il y a de nombres. Il suffit pour s'en convaincre de faire correspondre à chaque nombre son double. Cette correspondance est bien univoque dans les deux sens. Mais que deviennent alors les nombres impairs dans ce décompte? On voit ici l'une des particularités de l'infini: un ensemble infini peut avoir exactement autant d'éléments que l'un de ses sous-ensembles. Donc si de l'ensemble de tous les nombres on enlève les nombres pairs, il reste autant d'éléments dans l'ensemble qu'il y en avait au départ. On peut évoquer brièvement ici l'exemple que David Hilbert (1862-1943) aimait à utiliser dans ses cours :

Supposons un hôtel avec un nombre infini de chambres, toutes occupées. Néanmoins, même complet, il pourra accueillir un nouveau voyageur. Il suffit pour cela que chaque occupant d'une chambre passe dans la suivante. La première chambre est ainsi libérée. Et ainsi de suite.

Cantor utilise cette particularité dans la définition d'un ensemble infini (donnée ici dans la formulation de Dedekind):

\footnotetext{
«Un ensemble est infini s'il existe une correspondance univoque dans les deux sens entre cet ensemble et l'un de ses sous-ensembles propres », autrement dit s'il possède exactement autant d'éléments que l'une de ses parties propres.
}

Cette définition rend visible l'un des mécanismes fondamentaux de l'infini qui pourtant semble contraire à notre intuition. Cantor utilise précisément cette particularité pour caractériser le concept mathématique de l'infini, ou plutôt du transfini dans sa terminologie. Il reste à établir le rapport entre nombre et ensemble. Le plus simple est d'utiliser une approche dont l'idée remonte à Giuseppe Peano (1858-1932): Chaque nombre ordinal est (ou: correspond à) l'ensemble des nombres plus petits que lui. Comme le zéro est le plus petit nombre (naturel), il correspond à l'ensemble vide. Le un par contre correspond à l'ensemble qui contient le zéro, c'est-à-dire qui contient l'ensemble vide. Et ainsi de suite. L'ensemble qui contient tous les éléments d'un autre ensemble plus cet ensemble luimême est appelé ensemble successeur de ce dernier. Le successeur d'un nombre, plus exactement de l'ensemble correspondant à ce nombre, est donc bien, dans le cas fini, le nombre augmenté d'une unité et correspond à notre idée intuitive de «successeur». Si nous appliquons cette procédure dans le cas infini, c'est-à-dire si l'on considère l'ensemble de tous les nombres, nous pouvons le considérer comme premier nombre ordinal infini. Cantor lui a donné le nom de $\omega$. Ce nombre infini possède un successeur, dans le sens exact de notre définition du terme successeur, que l'on peut noter $\omega+1$. Cela signifie que $\omega$ ne représente qu'une 
première étape dans la structure de l'infini. Si l'on revient à la nouvelle de Kafka, on peut y voir métaphoriquement le premier gardien de l'infini. Mais puisque il existe un nombre $\omega+1$, il existe également un nombre $\omega+2$, un nombre $\omega+3$ et ainsi de suite. Puis un nombre $\omega+\omega$, et un nombre encore plus grand, $\omega \cdot \omega$. Egalement un nombre $\omega^{\omega}$. bref une suite sans fin de nombres construits à l'aide de $\omega$ et des opérations d'addition, de multiplication et d'exponentiation.

Tous les nombres ainsi obtenus à partir du nombre infini $\omega$, sont néanmoins dénombrables, c'est-à-dire qu'ils ont tous «le même nombre d'éléments », autrement dit, le même cardinal. Cantor nomme ce cardinal $\aleph_{0}$, en choisissant dans l'alphabet hébreu la première lettre, l'aleph, symboliquement proche dans la culture juive de la notion de Dieu et de l'infini. Or il apparaît que la suite des nombres $\left\{0,1,2, \ldots \omega, \ldots, \omega \cdot \omega, \ldots \omega^{\omega}, \ldots\right\}$, qui représente elle aussi un nombre ordinal, n'est plus dénombrable! Il s'agit donc d'un nombre transfini plus grand que $\aleph_{0}$. Ce premier nombre ordinal transfini dont la cardinalité dépasse $\aleph_{0}$ est transcrit dans la notation de Cantor par $\aleph_{1}$. Cela forme le début de la série illimitée des nombres cardinaux $\left\{\aleph_{0}, \aleph_{1}, \aleph_{2}, \ldots\right\}$ qui décrivent les différentes profondeurs de l'infini.

Il existe une forme d'arithmétique de ces nombres, qui ne possède pas les mêmes règles que l'arithmétique habituelle. On a par exemple $\aleph_{0}+\aleph_{0}=\aleph_{0}$ ainsi que $\aleph_{0} \cdot \aleph_{0}=\aleph_{0}$ ce qui signifie que par addition et multiplication on ne peut pas dépasser le stade du cardinal $\aleph_{0}$. Par contre, l'opération qui consiste à passer d'un ensemble à l'ensemble de toutes ses parties nous permet de dépasser la limite $\aleph_{0}$, c'est-à-dire que $2^{\kappa 0}>\boldsymbol{\aleph}_{0}$. Il y a donc des nombres cardinaux de plus en plus grands. Mais surtout, il ne peut pas exister de plus grand cardinal, ni de plus grand ordinal, c'est-à-dire que la suite des nombres transfinis ne peut pas être pensée comme une unité en termes d'ensemble.

Cantor se trouve devant l'infini comme l'homme de la campagne devant la loi, confronté à un édifice dont la complexité le dépasse. Un premier problème qui le préoccupe est celui de montrer que $\aleph_{0}$ est bien le plus petit des cardinaux transfinis. Il s'avère que pour montrer cela Cantor doit avoir recours à un principe nouveau, connu depuis sous le terme d'axiome du choix. Ce principe stipule que dans toute collection d'ensembles non vides, on peut former un nouvel ensemble qui contient exactement un élément de chaque ensemble de la collection de départ. L'axiome du choix semble innocent, voire totalement évident, mais son application est à l'origine d'un certain nombre de paradoxes, notamment topologiques, qui semblent contredire très sérieusement le bon sens commun. En particulier il permet de définir le découpage d'une sphère en un nombre fini de parties qui, une fois recomposées, forment deux sphères de même volume que la sphère d'origine (paradoxe de Banach-Tarski). La plausibilité de l'axiome du choix, et le problème de sa pertinence dans la pratique mathématique, est un premier obstacle sur lequel bute Cantor.

Si les nombres naturels sont de cardinalité $\aleph_{0}$, c'est-à-dire qu'il y a exactement $\aleph_{0}$ nombres de ce type, combien y a-t-il de nombres réels? Les nombres réels, c'est-à-dire les nombres qu'on peut écrire avec un développement (éventuellement infini) après la virgule, sont pour la plupart des mathématiciens l'image, le modèle du continu. A quelle profondeur de l'infini se situent les nombres réels? Or cette question du cardinal du continu est la deuxième barrière qui se dresse entre Cantor et l'infini et elle lui sera fatale. Cantor réussit à montrer par une magnifique démonstration que le cardinal de l'ensemble des nombres réels est 
donné par la formule $2^{\aleph o}$. Il y a donc autant de nombres réels qu'il y a de parties de nombres naturels! Mais la question qui hante Cantor est celle de savoir où se situe ce cardinal dans la hiérarchie de ses cardinaux transfinis $\left\{\aleph_{0}, \aleph_{1}, \aleph_{2}, \ldots\right\}$, ces gardiens successifs de l'infini. Il commence par l'égalité $2^{\aleph 0}=\aleph_{1}$ qui lui paraît évidente et qu'il espère démontrer facilement. Cela échoue à toutes ses tentatives. La question, connue depuis sous le terme d'hypothèse du continu, l'un des problèmes fondamentaux des mathématiques, l'obsède à tel point qu'il en devient malade ${ }^{9}$ et fera par la suite de nombreux séjours en clinique. Au cours de l'année 1884 par exemple, il écrit tour à tour à son ami Mittag-Leffler qu'il a trouvé une preuve simple de cette égalité, puis un peu plus tard, qu'il y a une erreur dans sa démonstration, puis plus tard encore, que son hypothèse est fausse. Il le dit en ces mots :

Und als ich in diesen Tagen wieder mich um denselben Zweck abmühte, da fand ich was? Ich fand einen strengen Beweis dafür, dass das Continuum nicht die Mächtigkeit der zweiten Z[ahlen]. cl[asse]. und noch mehr, dass es überhaupt keine durch eine Zahl angebbare Mächtigkeit hat ${ }^{10}$.

Cantor est totalement déstabilisé. De plus, sa théorie de l'infini est la cible d'attaques de nombreux collègues, de moins en moins rassurés par les aspects contre intuitifs et vertigineux de celle-ci. En particulier l'influent Kronecker, qui avait une position dominante à Berlin, essaie par tous les moyens d'empêcher la diffusion des idées de Cantor. En France également, l'opposition se manifeste, notamment par la voix de Poincaré, qui parle de la théorie de Cantor comme d'une maladie, dont les mathématiques allaient guérir un jour. Cantor se décourage, se désintéresse des mathématiques et se tourne vers la théologie et l'histoire de la littérature. Tout en ne quittant pas la «porte» de l'infini mathématique, il se concentre sur des détails sans rapport avec sa quête principale. Il essaie par exemple de montrer que Shakespeare n'est pas l'auteur des œuvres qu'on lui attribue, mais que celles-ci sont dues à Francis Bacon. Il écrit des opuscules théologiques sans grand intérêt, mais où l'infini et les mathématiques ne sont pas totalement absents. Il donne des cours de philosophie, sur Leibniz notamment, devant des auditoires de plus en plus limités ${ }^{11}$. Il se comporte comme l'homme de la campagne devant la loi, en se désintéressant apparemment de son but mais en restant obstinément figé devant l'obstacle. Vers la fin de sa vie, Cantor reprend ses travaux mathématiques. En 1891 il publie un bref article: Ueber eine elementare Frage zur Mannigfaltigkeitslehre ${ }^{12}$, sur la non-dénombrabilité des nombres réels. Puis quelques textes sur la théorie des ensembles. Mais ces derniers sont caractérisés par des lacunes qui ne seront comblées que par ses successeurs et surtout par son incapacité de résoudre le problème du continu, qui l'obsède jusqu'à sa mort, survenue à la clinique psychiatrique de Halle en 1916.

9 Les difficultés rencontrées dans ses tentatives ne sont probablement pas la seule cause de sa maladie, sa première dépression nerveuse, mais semblent y avoir contribué de manière essentielle.

10 Lettre du 14.11.1884

${ }^{11}$ «...allmählich schrumpfte die Zuhörerzahl auf 4, danach auf 3, dann 2 und schliesslich einen.» Lettre de Sonja Kowalewskaja à Mittag-Leffler du 21.5.1885.

12 Dans Jahresbericht der Deutschen Mathematiker Vereinigung 1. 
Ce problème se révèlera bien plus étrange et complexe que ne le soupçonne Cantor à la fin de sa vie. Ce n'est qu'en $1937^{13}$ que le logicien Kurt Gödel (19051978) découvre que l'hypothèse du continu, ainsi que l'axiome du choix, sont compatibles avec le reste de la théorie des ensembles, qui entre-temps avait été axiomatisée. Ce qui signifie qu'on ne peut pas prouver que l'hypothèse du continu est fausse. Etrange réponse, mais qui pouvait néanmoins encourager les chercheurs à tenter de prouver la vérité de cette hypothèse.

Le deuxième volet, le volet définitif, de la réponse apparaît au printemps 1963, lorsque Paul Cohen (1934- ), un très jeune mathématicien réussit le coup de force de démontrer, à l'aide d'une méthode raffinée qu'il avait inventée à cet effet, le forcing, que la négation de l'hypothèse du continu était elle aussi compatible avec la théorie axiomatique des ensembles, de même que la négation de l'axiome du choix. L'hypothèse du continu est donc indépendante des autres axiomes de la théorie des ensembles et le problème qui tracassait tant Cantor n'est pas décidable. L'hypothèse du continu n'est ni vraie ni fausse et toute activité de recherche a cessé autour de cette question, qui a préoccupé Cantor pendant des années et qui probablement a eu un effet négatif sur son état mental.

On peut imaginer que cette réponse aurait fait dans l'esprit de Cantor - s'il en avait eu connaissance - le même effet que vraisemblablement l'annonce finale du gardien a provoqué chez l'homme de la campagne dans la parabole de Kafka:

Ici nul autre que toi ne pouvait pénétrer, car cette entrée n'était faite que pour toi. Maintenant, je m'en vais et je ferme la porte.

Cela pourrait être la réaction du monde mathématique devant tout problème indécidable. L'indépendance du cinquième postulat d'Euclide en est probablement le premier exemple historique: après des siècles d'efforts acharnés pour montrer que ce postulat était vrai, ou faux, dans le cadre de la géométrie, il s'est avéré au cours du $\mathrm{XIX}^{\mathrm{e}}$ siècle, qu'on ne pouvait pas répondre à cette question ni par l'affirmative, ni par la négative. L'univers géométrique se scindait en plusieurs mondes parallèles, dont la géométrie Euclidienne n'était plus que l'un des volets. La question de départ, dans sa formulation naïve, n'avait plus de sens. Une porte s'était fermée, qui rendait apparemment inutiles tous les efforts précédents pour la résoudre. Pourtant si la quête d'une solution de ce problème n'a pas abouti, et ne pouvait pas aboutir, elle porte en elle-même sa signification, puisqu'elle a permis le développement des géométries non Euclidiennes. Comme dans le cas de Cantor, et finalement dans la nouvelle de Kafka, le sens de la quête n'est peut-être pas à chercher ailleurs que dans la quête elle-même.

La lecture du texte que nous avons proposée, est celle d'un mathématicien attentif aux structures suggérées dans la nouvelle, en particulier celle qui se rattache à la notion d'infini. Sans vouloir donner un sens précis à un texte qui ne peut pas en avoir qu'un seul, comme le montre très bien la scène de l'exégèse,

${ }^{13}$ Dans la nuit du 14 au 15 juin d'après des notes de Gödel lui-même. 
nous avons pris la nouvelle comme point de départ pour explorer des analogies, des coïncidences trouvées au travers de l'histoire de l'infini. Et l'attitude de Cantor devant l'infini - en particulier devant le problème de l'hypothèse du continu - nous a paru montrer une parenté intéressante avec le comportement de l'homme de la campagne, jusque dans son dénouement. Nous avons tenté une vision libre et arbitraire qui ne rend pas justice au texte concis de Kafka, mais qui prolonge les interrogations esquissées dans la scène du dôme dans une direction particulière, celle de la fascination de l'infini.

Université de Lausanne

\section{Quelques références}

[A] Amir D. Aczel, The mystery of the Aleph, Pocket Books 2000.

[C] Brian Clegg, A Brief History of Infinity, Robinson 2003.

[K] Robert \& Ellen Kaplan, The art of the infinite, our lost language of numbers, Penguin 2003.

[L] Tony Lévy, Figures de l' infini - Les mathématiques au miroir des cultures, Seuil 1987.

[R] Rudy Rucker, Infinity and the Mind - The science and philosphy of the infinite, Bantam Books 1982. 